\title{
5. Die Instrumente der Arbeitskräftelenkung
}

\section{Zwischen- und überbezirklicher Ausgleich}

Vor allem in den ersten Nachkriegsjahren war der zwischen- und überbezirkliche Ausgleich ein wesentliches Instrument, mit dem die Arbeitsverwaltung in der SBZ ein regionales Überangebot an Arbeitskräften rasch erkennen und in andere Gebiete weiterleiten wollte. Bedarfsgerechte Planung und Lenkung war das Ziel, das dahinter stand. Darüber hinaus sollte dieses Instrument einen Beitrag dazu liefern, regional entstandene oder sich ausbreitende Arbeitslosigkeit wieder abzubauen. Die Verwirklichung dieses arbeitsmarktpolitischen Ansatzes war in den ersten Nachkriegsjahren vor allem daran gescheitert, daß eine einheitliche Erfassung des Arbeitskräftepotentials, aufgegliedert nach einzelnen Berufssparten und Alterskohorten, regional differenziert, und dem jeweiligen Arbeitskräftebedarf gegenübergestellt, noch nicht möglich war. Dies hing, wie beschrieben, mit der anfänglichen Schwäche der Berliner Zentralverwaltung gegenüber den Landesverwaltungen, aber auch mit dem gemächlichen Aufbau der Arbeitskräfteregistrierung zusammen. Darüber hinaus muß in Rechnung gestellt werden, daß die Binnenwanderung von den Arbeitsämtern nicht gesteuert werden konnte.

Die ZVAS-Abteilung II versuchte frühzeitig das Verfahren dieses Arbeitskräfteausgleichs exakt festzulegen und gab am 6. November 1945 eine Verfügung heraus, die an alle Landesarbeitsämter verschickt wurde. Bei der gegenwärtigen Beschäftigungslage werde es - so die Berliner Zentralverwaltung - darum gehen, "die Vermittlungsaufträge in den Ausgleich zu geben, die wegen Fehlens der erforderlichen Arbeitskräfte im eigenen Bezirk nicht abgedeckt werden können"1. Jedes Arbeitsamt habe zunächst die eingehenden Vermittlungsaufträge „auf [ihre] unbedingte Notwendigkeit und den Grad der Dringlichkeit zu prüfen." Erst wenn die Prüfung ergeben habe, daß der Auftrag ,in angemessener Frist im eigenen Bezirk nicht abgedeckt werden kann", sei der zwischenbezirkliche Ausgleich "unverzüglich" einzuleiten. Im einzelnen hatten die einen zwischenbezirklichen Ausgleich beantragenden Arbeitsämter ein genaues Profil des zu besetzenden Arbeitsplatzes zu erstellen und dem abgebenden Arbeitsamt mitzuteilen. Gleichzeitig mußte auch gewährleistet sein, daß die zugewiesenen Arbeitskräfte für einen Ausgleich geeignet waren. Auf diese Weise sollte offensichtlich verhindert werden, daß der zwischenbezirkliche Ausgleich zu einem Verschiebebahnhof für nicht vermittelbare Arbeitsuchende mutierte. Schließlich war nach den Vorstellungen der ZVAS die ausreichende Versorgung der zugewiesenen Arbeiter mit Wohnraum und Lebensmitteln abzusichern. Bei einer Besprechung am 27. Februar 1946 stellte der stellvertretende Chef der SMAD-Abteilung Arbeitskraft, P. J. Morenow, die Veröffentlichung eines Befehls durch den Obersten Chef der SMAD, Georgi K. Schukow, in Aussicht, „der dem Präsidenten der [ZVAS] Vollmachten gibt, von sich aus diesen Ausgleich der Arbeitskräfte vorzunehmen“2. Dieser kam letztlich aber in dieser Form nicht zustande. 
Die ZVAS wollte mit der Verfügung den einzelnen Arbeitsämtern mehr Handlungsspielraum gewähren, hatte aber nicht damit gerechnet, daß sich diese oftmals direkt an die Berliner Zentralverwaltung wandten, was letztlich zu einer enormen Arbeitsüberlastung führte. So bat die ZVAS etwa das Provinzialamt für Arbeit und Sozialfürsorge in Potsdam am 31. Mai 1946, durch ein Rundschreiben die brandenburgischen Arbeitsämter dahingehend zu informieren, „daß sie, bevor sie den Reichsausgleich [sic] in Anspruch nehmen können, in allen Fällen erst einmal die Abdeckung der Anforderungen auf dem Wege der zwischenbezirklichen Vermittlung über das Provinzialamt für Arbeit und Sozialfürsorge in Potsdam erstreben müssen" 3 . Die ZVAS betonte auch gegenüber Vertretern der sowjetischen $\mathrm{Be}$ satzungsmacht, daß sie nur eingreifen werde, wenn ein Landesarbeitsamt den überbezirklichen Ausgleich in Berlin beantrage. Grundsätzlich bleibe die Arbeitsvermittlung jedoch Sache der Arbeitsämter bzw. Landesarbeitsämter ${ }^{4}$. Um die überregionale Vermittlung von Facharbeitern zu organisieren, schlug die ZVAS die Veröffentlichung von entsprechenden Stellengesuchen in der amtlichen Zeitschrift ,Arbeit und Sozialfürsorge" vor. Dies bezog sich allerdings ausdrücklich nur auf hochqualifiziertes Fachpersonal und nicht auf „allgemeine Berufe, für die allerorts Bedarf besteht, wie etwa Landarbeiter, Bauarbeiter, Metallarbeiter u. a." ${ }^{5}$.

Das Instrument des zwischenbezirklichen Ausgleichs erwies sich schon bald als fehlerhaft, da es auf seiten der Arbeitsämter eine Informationsdichte, -verarbeitung und -weitergabe voraussetzte, die in dem Umfang nicht zu leisten war. Darüber hinaus konnten auch auf diese Weise Fehlvermittlungen nicht ausgeschlossen werden, über deren Finanzierung im Frühjahr 1948 eine heftige Debatte zwischen den Landesverwaltungen und der DVAS/HVAS entbrannte. Unterschiedliche Meinungen bestanden schon bei der Frage der statistischen Erfassung des Arbeitskräfteausgleichs. So vertrat das thüringische Ministerium für Arbeit und Sozialwesen die Auffassung, daß sowohl beim zwischen- als auch beim überbezirklichen Ausgleich die Vermittlungen vom aufnehmenden Arbeitsamt zu zählen seien $^{6}$. Die Abteilung I a der DVAS bezog dagegen die entgegengesetzte Position und plädierte dafür, eine zustande gekommene Vermittlung durch das Arbeitsamt registrieren zu lassen, das die angeforderte Arbeitskraft gestellt hatte ${ }^{7}$. Diesem Standpunkt schloß sich auf Anfrage das Ministerium für Sozialwesen in Mecklenburg-Vorpommern an ${ }^{8}$. Die Berliner Zentralverwaltung hat jedoch allem Anschein nach eine Grundsatzentscheidung vermieden, so daß jede Landesverwaltung weiterhin die Modalitäten der Erfassung bestimmen konnte. Die Auseinandersetzung um die Erstattung der Kosten, die bei einer Fehlvermittlung entstanden, eskalierte erst, nachdem die Zahl dieser Fehlvermittlungen enorm angestie-

$3 \mathrm{BAB}, \mathrm{DQ} 2 / 2066$.

4 BAB, DQ 2/2040, Bl. 16, Aktenvermerk der DVAS-Abt. II über eine Besprechung mit der SMADAbt. Arbeitskraft am 1.6. 1946.

5 BAB, DQ 2/1738, Notiz der DVAS-Abt. II vom 1. 7. 1946.

- BAB, DQ 2/2195, Ministerium für Arbeit und Sozialwesen (HA Arbeit und Sozialfürsorge) des Landes Thüringen am 27. 1. 1948 an die DVAS.

ᄀ Ebenda, Leiter der Abt. I a (Donau) am 6. 2. 1948 an die Landesregierungen Sachsen, Sachsen-Anhalt, Brandenburg und Mecklenburg-Vorpommern.

8 Ebenda, Ministerium für Sozialwesen (HA Arbeit) der Landesregierung Mecklenburg-Vorpommern am 16. 2. 1948 an die DVAS-Abt. I a. 
gen war und die Länderhaushalte zunehmend belastete. Rechtliche Grundlage für das Erstattungsverfahren waren interessanterweise Bestimmungen aus der Zeit vor 1945: Zum einen die $\mathbb{\$} 132$ bis 135 und 140 des Gesetzes über Arbeitsvermittlung und Arbeitslosenversicherung (AVAVG) vom 16. Juli 1927 sowie die Richtlinien zur Förderung der Arbeitsaufnahme vom 22. März 1938. Die Arbeitsämter übernahmen nach Ende des Zweiten Weltkrieges dieses Verfahren „aus Gepflogenheit" und beglichen die angefallenen Kosten aus den Etatmitteln der Länder bzw. Provinzen'. Im Zusammenhang mit den steigenden Arbeitskräfteforderungen für den Erzbergbau mehrten sich die Streitfälle zwischen einzelnen Ländern über die weitere Kostenerstattung. Die Juristische Abteilung der HVAS bestätigte den Fortbestand der genannten rechtlichen Bestimmungen und verwies darauf, daß Schadensersatzansprüche "gegen Vermittler, die schuldhaft Fehlvermittlungen verursacht haben, $[\ldots]$ möglich" seien ${ }^{10}$. Eine grundlegende Veränderung der Kostenregelung war jedoch nicht zu erwarten und auch die Länder konnten sich keine Hoffnungen machen, bei der Finanzierung entscheidend entlastet zu werden.

Zusammenfassend muß hervorgehoben werden, daß der zwischen- und überbezirkliche Ausgleich die Erwartungen der Arbeitsverwaltung nie erfüllte. Die HVAS versuchte zwar in der Folgezeit, eine entsprechende Organisationsstruktur mit einzelnen Landesarbeitsämtern und den ihnen unterstehenden Arbeitsämtern aufzubauen. So sollten etwa in Mecklenburg-Vorpommern „Leitarbeitsämter" errichtet werden, denen einige Arbeitsämter zugeordnet werden sollten. Dadurch erhofften sich alle Beteiligten eine „schnellere Besetzung“ der offenen Stellen. Erst wenn die Leitarbeitsämter dazu nicht in der Lage waren, sollte nach den Planungen das Landesarbeitsamt eingeschaltet werden, „um über den Landesausgleich die Möglichkeit der Besetzung offener Stellen herbeizuführen"11. Eine Verbesserung des Arbeitskräfteausgleichs war allerdings auch dadurch nicht zu erreichen.

\section{Umschulung}

Die Arbeitsverwaltung griff bereits frühzeitig auf ein weiteres Instrument zurück, um vor allem die Nachfrage nach qualifizierten Arbeitskräften befriedigen zu können. Gerade auf diesem Gebiet hatte sich der zwischen- und überbezirkliche Ausgleich als weitgehend unzureichend erwiesen. Darüber hinaus waren die Nachteile des berufsfremden Arbeitseinsatzes ebenfalls deutlich geworden. Einen Anstoß gab die SMAD Anfang 1946, als sie darauf verwies, daß die Arbeitsämter sich verstärkt dieser Frage zuwenden sollten. Karlshorst erhoffte sich auf diese Weise, nicht nur dem Facharbeitermangel begegnen, sondern auch die Arbeitslosigkeit wirksam bekämpfen zu können ${ }^{12}$. Dafür sei es notwendig, so ein SMADVertreter, den Landesarbeits- und Arbeitsämtern „entsprechende Anweisungen

$9 \mathrm{BAB}, \mathrm{DQ} 2 / 111$, Bl. 154, HVAS-Abt. I b (Donau) am 7. 9. 1948 an die Juristische Abt.

10 Ebenda, Bl. 156, Juristische Abt. am 13. 9. 1948 an die Abt. I b.

$11 \mathrm{BAB}, \mathrm{DQ} 2 / 2063$, Bericht der HVAS (Abt. I a) über die Amtsleitertagung am 5./6. 3. 1949 in Ludwigslust, S. 3.

$12 \mathrm{BAB}, \mathrm{DQ}$ 2/1, Bl. 38, Aktenvermerk über Besprechung mit Remissow (SMAD) am 13. 2. 1946. 
zu geben und eine fortgesetzt ernste Kontrolle durchzuführen." Zwei Wochen später wiederholte die SMAD-Abteilung Arbeitskraft ihren Vorschlag13. Anfang Mai wurde die Veröffentlichung eines sowjetischen Befehls „über Umschulung und Ausbildung der Arbeitskräfte" in Aussicht gestellt ${ }^{14}$. Mit dem Befehl Nr. 140 über die „Ausbildung von qualifizierten Arbeitskräften und Umschulung von Arbeitskräften " beauftragte die SMAD den DVAS-Präsidenten zunächst einmal, den Facharbeiterbedarf, getrennt nach den wichtigsten Industriezweigen, festzustellen ${ }^{15}$. Außerdem erhielt die Berliner Zentralverwaltung die Aufgabe, gemeinsam mit den Präsidenten der Landes- bzw. Provinzialverwaltungen dafür zu sorgen, daß „kurzfristige Anlern- und Umschulungskurse im Jahr 1946 für Arbeitskräfte in den Mangelberufen" eingerichtet werden. Das Gesamtkontingent belief sich auf insgesamt 180000 Personen: 60000 in Sachsen, 50000 in Sachsen-Anhalt, 35000 in Thüringen, 23000 in Brandenburg und 12000 in Mecklenburg-Vorpommern ${ }^{16}$.

Bei der Realisierung des Umschulungsprogramms ergaben sich einige Schwierigkeiten, die auf eine mangelnde Absprache zwischen sowjetischen und deutschen Stellen hindeuteten. So registrierte die DVAS am 8. Juli 1946, daß die sächsische Landesregierung einen Umschulungsplan aufgestellt hatte, der nur 30000 Umschüler vorsah ${ }^{17}$. Bis zum 31. Mai waren dort bereits 18950 Personen eingesetzt worden, vornehmlich im Baugewerbe (11200) und in der Metallindustrie (3050). Der weitere Ablauf des Programms wurde allerdings dadurch behindert, $\mathrm{da} ß$ die in Sachsen im März wieder einsetzende Demontage gerade die Betriebe traf, die zur Ausbildung der Umschüler vorgesehen waren. Darüber hinaus konnte die Landesverwaltung die von der SMAD vorgegebene Zahl von 60000 Umschülern nicht erreichen: „Für die Anlernung von 60000 Personen [...] stehen im wesentlichen nur Frauen und Jugendliche (Schulabgänger) zur Verfügung." 18 Damit hatte sich das eigentliche Ziel des Befehls langsam gewandelt: von der Umschulung bereits Berufstätiger hin zur Gewinnung neuer Arbeitskräfte. Die Lehrlingsausbildung rückte immer stärker in den Mittelpunkt des Interesses. Als weitere Bevölkerungsgruppe kamen auch die Flüchtlinge und Vertriebenen in Betracht: Die Zentralverwaltung für deutsche Umsiedler (ZVU) forderte die Landes- und Provinzialverwaltungen auf, „sich dafür einzusetzen, daß die Umsiedler in erster Linie Berücksichtigung finden" 19 .

Bis zum Jahresende 1946 hatte sich die Lage deutlich verbessert. So berichtete etwa das thüringische Landesamt für Arbeit und Sozialfürsorge, daß bereits 28292 Arbeitskräfte eine kurzfristige Umschulungsmaßnahme absolviert hätten, während sich weitere 13950 noch in einem der zahlreichen Umschulungskurse befän-

13 Ebenda, Bl. 44, Aktenvermerk über Besprechung mit Morenow (SMAD) am 27. 2. 1946.

14 Ebenda, Bl. 70, Aktenvermerk über Besprechung mit Morenow und Lamin (SMAD) am 10. 5. 1946.

15 SMAD-Befehl Nr. 140 vom 10. 5. 1946, in: Arbeit und Sozialfürsorge 1 (1946), S. 149f. Der Befehl wurde sehr wahrscheinlich erst einige Tage später den deutschen Stellen übergeben.

16 Ebenda, S. 150.

$17 \mathrm{BAB}, \mathrm{DQ} 2 / 797$, Notiz der HVAS vom 8. 7. 1949, S. 2.

18 Ebenda.

$19 \mathrm{BAB}, \mathrm{DO} 2 / 59, \mathrm{Bl} .396, \mathrm{ZVU}$ am 20. 5. 1946 an die Landes- und Provinzialverwaltungen. 
den20. Damit konnte das Landesamt Thüringen insgesamt 42242 Umschüler vorweisen - bei einer vorgegebenen Sollgröße in Höhe von 35000 ein beachtlicher Erfolg. Der SMAD-Befehl Nr. 140 wurde jedoch keineswegs als erfüllt angesehen. Vielmehr diene - so das Landesamt in Weimar - die „Anlernung der für Thüringen vorgesehenen $35000 \mathrm{Kräfte}$ in den einzelnen Mangelberufen dazu, in naher Zukunft eine Arbeitskräftereserve für den Aufbau Deutschlands zu bilden “21. Ein abschließendes Urteil über Erfolg oder Mißerfolg dieser Aktion kann jedoch erst gefällt werden, wenn die Aufteilung der Umschüler auf die einzelnen Wirtschaftsbranchen untersucht wird. Gelang es also, den Bedarf für die Grundstoff- und Schwerindustrie abzudecken? Die Antwort fällt weniger eindeutig aus: In Thüringen befanden sich zahlreiche Umschüler in der Land- und Forstwirtschaft, obwohl die SMAD für diese Bereiche keine Auflagen gemacht hatte. Und weiter: Während für einzelne Industriezweige die Auflage übertroffen werden konnte (z.B. Bergbau, Metall- und Textilindustrie), wiesen andere Bereiche einen Fehlbestand auf (vor allem die besonders wichtige Bauindustrie)22. Die Abteilung Arbeit und Sozialfürsorge des SED-Zentralsekretariats, die im Dezember 1946 die Tätigkeit der SED-Landesleitungen untersuchte, kritisierte die Durchführung des SMAD-Befehls Nr. 140 in Thüringen. Die Umschulung lasse vor allem im Hinblick auf die Arbeitskräftelenkung „viel zu wünschen übrig“23. Die einzelnen Maßnahmen müßten stets mit dem Ziel verknüpft werden, neue Arbeitskräfte zu gewinnen und die Arbeitsproduktivität in den Betrieben zu steigern. Andernfalls seien - so die Mitarbeiter der SED-Führung aus Berlin weiter - „schließlich einmal wirklich keine verfügbaren Arbeitskräfte“ in Thüringen mehr vorhanden. Letztlich ließ sich das zuständige Landesamt bei der Umschulungsaktion vom Prinzip der Freiwilligkeit leiten. Unter Bezugnahme auf einen angeblichen Fall einer Zwangseinweisung eines Jugendlichen wurde ausdrücklich betont, es sei „kein einziger Fall bekannt geworden, daß in Thüringen bei der Durchkämmung von Banken Jugendliche in andere Betriebe gepreßt wurden"24. Diese Position vertraten im übrigen auch die DVAS sowie die anderen Landesverwaltungen, die sich aber in dem Dilemma befanden, die von der sowjetischen Besatzungsmacht erhobenen Auflagen dennoch erfüllen zu müssen.

Die Reichweite der beruflichen Umschulung blieb auch weiterhin begrenzt. So gelangte eine Abteilung des FDGB-Bundesvorstandes Anfang $1948 \mathrm{zu}$ der Schlußfolgerung, daß die von den Arbeitsämtern und den Betrieben durchgeführten Maßnahmen nicht ausreichen würden, um den Facharbeitermangel zu beheben $^{25}$. Die Industriegewerkschaften wurden aufgefordert, von sich aus Lehrgänge zur Fort- und Weiterbildung einzurichten. Die bisher gewonnenen Erfahrungen

$20 \mathrm{BAB}, \mathrm{DQ} 2 / 2075$, Bl. 45-55, hier Bl. 45, Bericht des thüringischen Landesamtes für Arbeit und Sozialfürsorge vom 1. 12. 1946.

21 Ebenda.

22 Ebenda, Bl. 46 und 55.

23 SAPMO, DY 30/IV 2/2.027/2, Bl. 25-27, hier Bl. 25, Bericht über Besprechungen betr. Arbeitslenkung in Weimar und Halle am 18. bzw. 20. 12. 1946.

24 SAPMO, DY 30/IV 2/16/108, Bl. 239, Land Thüringen, Ministerium für Wirtschaft, Arbeit und Verkehr am 3. 1. 1947 an die DVAS.

25 SAPMO, DY 34/20149, Bericht der Abt. Arbeitslenkung, Berufsausbildung, Kriegsgefangene, Heimkehrer, Umsiedler vom 11. 1. 1948, S. 2. 
veranlaßten die DVAS dazu, die betriebliche Berufsausbildung insgesamt zu stärken, und auf diese Weise den Arbeitskräftebedarf langfristig abzudecken zu versuchen. Es gab zwar für das Jahr 1948 einen Umschulungsplan, allerdings auf erheblich niedrigerem Niveau. So waren in Sachsen insgesamt 11750 Umschüler vorgesehen; die Auflage konnte zu etwa 80 Prozent erfüllt werden (9377) 26.

\section{Staatliche Beschäftigungsprogramme}

Erste Ansätze zu einer aktiven Beschäftigungspolitik kamen von seiten der SEDFührung erst 1948, wobei an dieser Stelle die von den Kommunalverwaltungen in Auftrag gegebenen Enttrümmerungs- und Aufräumarbeiten der unmittelbaren Nachkriegszeit weitgehend unberücksichtigt bleiben. Im Mittelpunkt stehen vielmehr Programme, mit denen die SBZ-Sozialpolitiker den Arbeitsmarkt direkt und positiv beeinflussen wollten. Eine Arbeitsgruppe des SED-Parteivorstandes hatte 1948 in einer internen Denkschrift darauf hingewiesen, daß die Neuordnung der Wirtschaft „eine gewisse Freistellung von Arbeitskräften ergeben“ habe27. Eine Bereitstellung von Arbeitsplätzen in größerem Umfange sei erst nach Genehmigung und Durchführung des Investitionsplanes zu erwarten. Als Sofortmaßnahme wurde im einzelnen vorgeschlagen, insgesamt 67000 Arbeitskräfte neu einzustellen: 30000 für Meliorationsarbeiten, 8000 für Aufforstungstätigkeiten, 8000 für den Bau neuer Wohnungen, 12000 für die Wiederherstellung von zerstörtem Wohnraum sowie 9000 für die Herstellung von Ziegeln. Das Kleine Sekretariat des SED-Politbüros stimmte diesem Sofortprogramm am 18. März 1949 $\mathrm{zu}^{28}$.

Finanzierungsfragen ergaben sich bei der Ausarbeitung der einzelnen kreditfinanzierten Beschäftigungsprogramme. Einzelne Landesverwaltungen sprachen sich für eine Verlagerung der Kosten auf die Sozialversicherung in Gestalt einer "wertschaffenden Arbeitslosenunterstützung " aus ${ }^{29}$ : Dies lief letztlich auf eine $\mathrm{Ar}$ beitsverpflichtung für Arbeitslose hinaus. Im Gegensatz zu den Landesregierungen drängte die HVAS auf eine spürbare Senkung der Gesamtkosten durch eine deutliche Reduzierung des monatlichen Durchschnitsslohnes ${ }^{30}$. Nach Angaben der HVAS waren die zuständigen Landesministerien "nicht in der Lage gewesen, auf die Produktionszahlen und die Zahl der anzufordernden Arbeitskräfte aufgrund der vorgesehenen Wirtschaftspläne zurückzugreifen, da diese Angaben noch nicht vorliegen" ${ }^{31}$. Die Durchführung der Sofortmaßnahmen zog sich damit in die Länge. Die Planungsarbeiten der Landesministerien wurden von der HVAS aufmerksam verfolgt. Besonders Mecklenburg mußte sich deutliche Kritik gefallen

$26 \mathrm{BAB}, \mathrm{DQ} 2 / 1763$, Protokoll über dic Amtsleitertagung am 13./14. 5. 1949 in Chemnitz, S. 23.

27 SAPMO, NY 4182/1158, Bl. 181-183, hier Bl. 181. Der Kommission gehörten Paul Wessel, Horst Paffrath, Straßenberger und Karl Litke an.

28 SAPMO, DY 30/J IV 2/3/13, Bl. 7 und $31 \mathrm{f}$.

$29 \mathrm{BAB}, \mathrm{DQ}$ 2/970, Minister für Arbeit und Sozialwesen der Landesregierung Brandenburg am 21. 2. 1949 an die HVAS.

30 SAPMO, DY 30/IV 2/2.027/21, Bl. 93-96, Bericht der HVAS vom 25. 2. 1949 an die Abt. Arbeitskraft der SMAD.

31 Ebenda, Bl. 94. 
lassen: Dort sei die Tätigkeit des Landesministeriums „völlig ungenügend“32. Obwohl im Januar 194939320 Personen Arbeit gesucht hätten, seien im Beschäftigungsprogramm der mecklenburgischen Landesregierung nur 7420 neue Stellen vorgesehen. Damit habe die Landesregierung die auf einer Konferenz der Arbeitsminister gestellten Aufgaben „nicht erfüllt.“ Die DWK-Hauptverwaltung Wirtschaftsplanung betrachtete die Freisetzung von Arbeitskräften aus einer völlig anderen, nämlich primär makroökonomischen Perspektive und gelangte daher auch zu ganz anderen Schlußfolgerungen: „Die Erscheinung, daß durch die Produktivitätssteigerung Arbeitskräfte frei werden, ist durchaus positiv und als Gesundungsmerkmal der Wirtschaft zu werten." Die Hauptverwaltung ging sogar noch weiter und verlangte die Fortsetzung dieses Prozesses, der „mit allen Mitteln voranzutreiben [sei] und nicht durch die Sorge um gegebenfalls freiwerdende Arbeitskräfte gehemmt werden" dürfe. Probleme bei der beruflichen Unterbringung der dabei wachsenden Gruppe der Erwerbslosen wurden von ihr nicht gesehen: Tätigkeiten seien vielmehr, ,im reichlichen Umfange vorhanden“"33. Somit standen sich zwei Ansätze gegenüber. Während die HVAS auf die rasche Beseitigung der Arbeitslosigkeit bedacht war, legte die Hauptverwaltung Wirtschaftsplanung den inhaltlichen Schwerpunkt auf die Steigerung der Produktivitätsraten vor allem in den volkseigenen Betrieben und sah in der dabei um sich greifenden Entlassung von Arbeitern nur ein temporäres Problem, das im Zuge der längerfristigen Wirtschaftsplanung zu lösen sei. Damit war bereits die Perspektive für den Zweijahrplan gelegt; doch zunächst hatte sich die Arbeitsverwaltung mit der weiteren Durchführung des Sofortprogrammes intensiv zu befassen.

Konkrete Umsetzungsschwierigkeiten tauchten rasch in den Ländern auf; dabei zeigten sich vor allem auch die nicht unbeträchtlichen regionalen Unterschiede. So meldete etwa das Amt für Arbeit und Sozialfürsorge in Potsdam, daß in der Stadt die Enttrümmerungsarbeiten ,in letzter Zeit einen Auftrieb genommen“ hätten, da „neue Gelder für die Durchführung dieser Arbeiten zur Verfügung gestellt" wurden ${ }^{34}$. Außerdem seien für den Bau einer Wasserleitung von Potsdam nach Babelsberg rund 110 Arbeiter und für die Kabelverlegung 120 Arbeiter neu eingestellt worden. Der Erfolg des Beschäftigungsprogramms hing mit der Situation auf dem Arbeitsmarkt unmittelbar zusammen: Während verschiedene Arbeitsämter in Brandenburg eine vergleichsweise hohe Arbeitslosenrate registrierten, war dies beispielsweise in Potsdam nicht der Fall. Somit standen in zahlreichen ländlichen Regionen sehr viel mehr Arbeitskräfte zur Verfügung, die für die einzelnen Teilprogramme eingesetzt werden konnten bzw. sich oftmals auch freiwillig meldeten. Dagegen bildeten die Städte eindeutig die Schwerpunkte des Beschäftigungsprogramms, da hier das Arbeitsplatzangebot aus Sicht der Arbeitsverwaltung ungleich schneller auszuweiten war, als dies auf dem Lande im gleichen quantitativen Maßstabe möglich gewesen wäre. Gleichzeitig kollidierten

32 SAPMO, DY 30/IV 2/2.027/21, Bl. 97-104, hier Bl. 99, Bericht des Leiters der HVAS vom 25. 2. 1949.

33 BAB, DC 15/63, Bl. 15f., Vermerk der DWK-HV Wirtschaftsplanung (HA Arbeitskräfte) vom 28. 2. 1949.

34 BAB, DQ 2/2063, Protokoll der Sitzung des Beratungsausschusses des Amtes für Arbeit und Sozialfürsorge Potsdam vom 7. 3. 1949. 
die städtischen arbeitsmarktpolitischen Initiativen mit den sowjetischen Anforderungen, ganz besonders mit den Befehlen zur Bereitstellung von Arbeitskräftekontingenten für die Wismut AG. Die Arbeitsämter mußten diesen Faktor stets als unberechenbare Variable einkalkulieren, hatten jedoch keinerlei Einfluß auf sie, sondern konnten darauf nur reagieren. Längerfristige Planungen auf anderen Teilarbeitsmärkten fanden daher unter äußerst erschwerten Bedingungen statt. So vertrat der Beratungsausschuß des Potsdamer Arbeitsamtes folgerichtig die Auffassung, daß die auferlegten Zahlen für den Erzbergbau zu hoch seien, und bat um eine deutliche Senkung der Anforderungen: „Wenn die Bereitstellung der Arbeitskräfte in der geforderten Höhe noch nicht erfolgen konnte, so liegt dies lediglich daran, daß diese Kräfte aus ihren Arbeitsstellen herausgezogen werden müßten. "35 Mit solchen Problemen hätten jedoch nicht die Arbeitsämter zu kämpfen, in deren „Bezirken Arbeitslose und Arbeitslosenunterstützungsempfänger vorhanden sind." Doch auch diese plausibel klingende Argumentation konnte offensichtlich die sowjetische Besatzungsmacht nicht davon überzeugen, in Zukunft die eigenen Arbeitskräfteanforderungen besser mit der deutschen Arbeitsverwaltung abzustimmen.

Die SED-Betriebsgruppe bei der DWK warnte am 5. April 1949 vor einer überhasteten und gedankenlosen Auskämmung von Betrieben für die neuen arbeitsmarktpolitischen Projekte: „Es wäre falsch, jetzt zu viele Textilarbeiter umzusetzen und umzuschulen und sie nach einem Jahr wieder in die Textilindustrie zurück zu führen. “36 Ähnlich wie die Hauptverwaltung Wirtschaftsplanung legte auch die SED-Betriebsgruppe den wirtschaftspolitischen Schwerpunkt ausschließlich auf die Steigerung der Produktivität in den Betrieben und vernachlässigte arbeitsmarktpolitische Ziele: „Das Freiwerden von Arbeitskräften durch Produktivitätssteigerung ist ein Gesundungsmerkmal und führt zur Rentabilität der volkseigenen Wirtschaft. Diese Entwicklung darf auf keinen Fall zu opportunistischen Einstellungen führen, die beispielsweise die Steigerung der Produktivität bremsen, um keine Arbeitskräfte freisetzen zu müssen. " ${ }^{37}$ Damit fehlten der HVAS wichtige Bündnispartner zur erfolgreichen Durchsetzung des Sofortprogramms, das immer mehr den Charakter einer Absichtserklärung gewann und keine durchschlagende Wirkung entfalten konnte, da für die wirtschaftlichen Hauptverwaltungen die Erfüllung der Wirtschaftspläne Vorrang genoß. Vorschläge von seiten der Hauptverwaltung Wirtschaftsplanung, zusätzliche Mittel zur Finanzierung des Sofortprogramms aus den Kassen der Sozialversicherungsanstalten abzuziehen, wehrte die HVAS brüsk ab, die sich verpflichtet fühlte, „die Kontrolle über die Verwendung der Mittel aus der Sozialversicherung strengstens auszuüben“"38. Die ursprünglich eingeplante Bereitstellung von 9,5 Millionen DM wurde sogar explizit in Frage gestellt, „wenn nicht sofort seitens der Wirtschaftsplanung der Länder und der Investitionsbank die Maßnahmen getroffen werden, daß die vorgesehenen rund 67000 Arbeitskräfte in Beschäftigung gebracht werden können.“

35 Ebenda.

36 BAB, DC 15/63, Bl. 17f., hier Bl. 17, Notiz der SED-Betriebsgruppe bei der DWK vom 5. 4. 1949.

37 Ebenda.

38 BAB, DQ 2/970, Bericht Litkes vom 27. 4. 1949. 
Die Anstrengungen der HVAS reichten allerdings nicht aus, um einen positiven Abschluß des Beschäftigungsprogramms vermelden zu können. So herrschte Ende April immer noch ein Planungschaos zwischen den Ländern und der zuständigen Berliner Hauptverwaltung ${ }^{39}$. Der stellvertretende Leiter der HVAS, Karl Litke, stellte unverblümt fest: „Unsere Pläne haben unsichtbare Mängel, es gibt aber sichtbare Lenkungsmängel." 40 Kurz darauf zog Litke etwas resigniert ein vorläufiges Resümee des abgelaufenen Beschäftigungsprogramms: „Diese Aktion war ein Fehlschlag. “41 Im Rahmen des Sofortprogramms I hätten 80900 Arbeitskräfte neu eingestellt werden sollen. Tatsächlich meldeten die Länder aber nur 20479 neue Beschäftigungsverhältnisse. Der führende Mitarbeiter der Berliner Hauptverwaltung sah den Hauptgrund für diesen Mißerfolg in der unzureichenden finanziellen Ausstattung der Landeshaushalte. Die Investitionsbank habe zudem die angeforderten Mittel nicht bereitgestellt. Für das geplante Sofortprogramm II, bei dem 115819 neue Arbeitsplätze vorgesehen waren, sprach Litke die Warnung aus, daß auch diese Aktion scheitern werde, „wenn nicht mit äußerster Energie an [die] Durchführung dieser Maßnahmen herangegangen wird“42. Er schlug vor, daß „lohnintensive Maßnahmen mit geringem Materialaufwand [...] am zweckmäßigsten" seien ${ }^{43}$. Sie würden allerdings keine grundlegende Kehrtwende auf dem Arbeitsmarkt bringen. Statt dessen müßten vielmehr Großprojekte durchgeführt werden, „sonst wird an Symptomen herumkuriert, wie der zurzeitige Stand des Sofortprogramms I ausweist.“

Nachdem erkennbar geworden war, daß sich die Sofortprogramme nicht in dem Maße erfolgreich durchführen ließen, wie sich das die SED-Führung, aber auch die Leitung der HVAS erhofft hatten, gerieten die weiteren Planungsarbeiten für beschäftigungspolitische Programme zunächst ins Stocken. Ein weiteres kam hinzu: Die sich zu diesem Zeitpunkt intensivierende allgemeine und zentrale Wirtschaftsplanung gewann immer stärker an Bedeutung; ihr wurden andere Ziele sukzessive untergeordnet. Das galt auch für die Sofortprogramme, die unter anderem zur Versorgung von Erwerbslosen mit Arbeit gedacht waren, während der anlaufende Zweijahrplan primär andere Ziele verfolgen sollte, nämlich die Steigerung der Arbeitsproduktivität in den Betrieben. Dieser Zielkonflikt wurde letztlich zugunsten der allgemeinen Wirtschaftsplanung entschieden; die HVAS hatte insofern das Nachsehen gegenüber der Hauptverwaltung Wirtschaftsplanung. Um dieses Ziel zu erreichen, wurde ein Ansteigen der Arbeitslosenzahlen, darauf ist bereits hingewiesen worden, in Kauf genommen. Aus Sicht der Arbeitsverwaltung ließ sich im übrigen auf diese Weise ein anderes Problem lösen, dem man bisher stets hilflos begegnet war: der Hortung von Arbeitskräften. Durch die Anweisung an die volkseigenen Betriebe sowie die SAG-Betriebe, die von seiten der SMAD bzw. der SMA in den Ländern eine entsprechende Anweisung erhielten, rentabel zu produzieren, „wurden alle bis dahin gehorteten Arbeitskräfte

39 SAPMO, DY 30/IV 2/2.027/21, Bl. 123-137, Bericht Karl Litkes vom 21. 4. 1949.

to Ebenda, Bl. 125.

41 SAPMO, DY 30/IV 2/2.027/21, Bl. 116-120, hier Bl. 116, Bericht der HVAS vom 29. 4. 1949 an die Abt. Arbeit und Sozialfürsorge beim SED-Politbüro.

42 Ebenda, Bl. 118.

43 Ebenda, Bl. 119. 
entlassen" ${ }^{44}$. Gleichzeitig mußten einige Arbeitsämter jedoch feststellen, daß die Betriebe „bei den Entlassungen nicht die besten Arbeitskräfte den Ämtern für Arbeit zur Verfügung" stellen. Das Arbeitsamt Zeitz gelangte zu der Schlußfolgerung: „Es bedarf noch einer großen Aufklärung seitens des FDGB, die Betriebsvertretungen sowie Betriebsleitungen zu überzeugen, daß es notwendig ist, wenn wir die Aufgaben des Zweijahrplanes erfüllen wollen, dem Arbeitsmarkt nur gesunde kräftige Leute, welche in ihren Betrieben abkömmlich sind bezw. umgesetzt werden können, zur Verfügung zu stellen.“ ${ }^{45}$ Knapp zwei Monate später mußte das Arbeitsamt Zeitz erneut melden, daß das Verständnis auf seiten der Betriebe, „gute Arbeitskräfte anderen Industriezweigen im Zuge des Zweijahrplans“ zuzuführen, „leider nicht vorhanden" sei $^{46}$. Offensichtlich erwies sich diese Hoffnung rasch als Illusion, da die Betriebe nicht bereit waren, Facharbeiter abzugeben. Darüber hinaus bestand nahezu in allen Wirtschaftszweigen der SBZ eine Übernachfrage nach qualifizierten Kräften. Der von den wirtschaftlichen Hauptverwaltungen bewertete "Gesundungsprozeß" hatte im Februar und März 1949 zu einem Ansteigen der Zahl der Empfänger von Arbeitslosenunterstützung sowie von Fürsorgeleistungen geführt ${ }^{47}$. Beides traf insbesondere Frauen. Festzuhalten bleibt, daß die Arbeitsämter in der Folgezeit aufgrund des geschilderten Richtungswechsels nur sehr begrenzt beschäftigungspolitische Initiativen starten konnten. Diese beschränkten sich im wesentlichen auf Enttrümmerungsarbeiten ${ }^{48}$.

Im Frühjahr 1949 wurden in der DWK erste Besprechungen zur Vorbereitung von "Großprojekten“" mit dem Ziel geführt, zahlreiche Arbeitsplätze für die Arbeitslosen in der SBZ anzubieten, deren Zahl im März 1949 auf 390000 angewachsen waren. Bereits Ende Mai sicherte das Kleine Sekretariat des Politbüros die Finanzierung des Sofortprogramms II durch einen entsprechenden Beschluß $\mathrm{ab}^{49}$. Demzufolge sollten insgesamt 207 Millionen DM zur Verfügung gestellt werden ${ }^{50}$. Nach Berechnungen der HVAS, die auf den Vorschlägen der Landesverwaltungen beruhten, waren insgesamt 229 Millionen DM erforderlich, um 115000 Personen eine Beschäftigung zu bieten ${ }^{51}$. Dieses Vorhaben firmierte bereits Ende Mai nicht mehr unter der Bezeichnung „Sofortprogramm II“, sondern als Zusatzplan zum Investitionsplan ${ }^{52}$. Auch dadurch war die Einbindung der Ar-

44 LA Magdeburg LHA, Rep. K MW, Nr. 10076, Bl. 52, Amt für Arbeit und Sozialfürsorge Zeitz am 11. 5. 1949 an den Minister für Arbeit und Sozialpolitik von Sachsen-Anhalt.

45 Ebenda.

46 LA Magdeburg LHA, Rep. K MW, Nr. 10076, Bl. 60, Amt für Arbeit und Sozialfürsorge Zeitz am 7. 7. 1949 an den Minister für Arbeit und Sozialpolitik von Sachsen-Anhalt.

$47 \mathrm{BAB}, \mathrm{DQ} 2 / 517$, Übersicht der HVAS über die Arbeitslosenunterstützungsempfänger in der SBZ (Dezember 1948-Juli 1949).

$48 \mathrm{BAB}, \mathrm{DQ} 2$ 2/1763, Protokoll über die Amtsleitertagung am 13./14. 5. 1949 in Chemnitz, S. 9 f.

49 SAPMO, DY 30/IV 2/2.027/21, Bl. 174, Protokoll der Sitzung des Kleinen Sekretariats vom 31. 5. 1949.

50 Im einzelnen waren 17 Millionen von den wasserwirtschaftlichen Verbänden, fünf Millionen aus den Sonderhaushalten der Länder und 185 Millionen aus dem Sperrfonds der Sozialversicherungsanstalten, der bedingt durch die Währungsumstellung eine Gesamthöhe von 400 Milliarden DM hatte, zu erbringen.

$51 \mathrm{BAB}, \mathrm{DQ} 2 / 1303, \mathrm{Bl} .12-23$, hier Bl. 13, Bericht der HVAS über die Arbeitsministerkonferenz am 31. 5. 1949 in Berlin (Ausführungen Bracks).

52 Ebenda, Bl. 14 (Ausführungen Litkes). 
beitsmarktpolitk in die allgemeine Wirtschaftsplanung deutlich geworden. Auf diese Weise wurde die Hauptverwaltung Wirtschaftsplanung weiter aufgewertet, die bei der Aufstellung der Arbeitskräftepläne immer wichtiger werden sollte. $\mathrm{Da}$ die DWK nunmehr dazu überging, für sämtliche Wirtschaftsbereiche Planungsziffern zu erstellen und auszugeben, war es nur zwangsläufig, daß der Faktoreinsatz ,Arbeit' von dieser Entwicklung nicht ausgenommen wurde. Die Protokolle der Arbeitsministerkonferenzen 1949, zu denen nun auch Vertreter der Hauptverwaltung Wirtschaftsplanung eingeladen wurden, enthalten interessanterweise keinerlei Hinweise auf Konflikte zwischen den beiden beteiligten Hauptverwaltungen. Die HVAS nahm diesen Kompetenzverlust offenbar ohne weiteres hin.

Bereits am 2. Juli 1949 legte die Hauptverwaltung Wirtschaftsplanung „Richtlinien für die Kontrolle des Einsatzes von Arbeitskräften in den Ländern der SBZ" vor, die sich davon einen umfassenderen Überblick über die bisherige Durchführung der Beschäftigungsprogramme im Zusammenhang mit dem Investitionsplan erhoffte ${ }^{53}$. Daher sollten bei den Kontrollen nicht nur der quantitative Umfang der erfolgten Neueinstellungen erfaßt, sondern auch die weiteren Aufnahmekapazitäten in den einzelnen Betrieben ermittelt werden. Darüber hinaus mußten nach den Vorstellungen der Hauptverwaltung Wirtschaftsplanung die bereits eingesetzten Investitionsmittel festgestellt werden. Die Überprüfungen, die zunächst nur in Brandenburg und Mecklenburg-Vorpommern durch die Kontrollabteilung der Hauptverwaltung Wirtschaftsplanung unter Beteiligung von zwei HVAS-Mitarbeitern durchgeführt werden sollten, hatten sich auf die zuständigen Verwaltungen auf Landes- und Kreisebene sowie auf die Betriebe zu erstrecken. Mit den Kontrollen in den übrigen Ländern wurden die Kontrollabteilungen der Hauptverwaltung Wirtschaftsplanung in den Ländern beauftragt. Diese Maßnahme erschien den politisch Verantwortlichen notwendig geworden zu sein, nachdem einzelne Arbeitsämter über die Erfolglosigkeit der Sofortprogramme geklagt hatten. Die angestrebte Entlastung auf dem Arbeitsmarkt war demzufolge nicht eingetreten, wobei dies auch auf unzureichende Absprachen zwischen der Hauptverwaltung Wirtschaftsplanung und der Investitionsbank zurückgeführt werden konnte. So wies etwa das Arbeitsamt Zeitz darauf hin, daß "die hiesige Kreisverwaltung beschränkte Mittel nur für bestimmte Arbeiten aus dem Fond der Investitionsbank erhalten hat, während die Anträge der Stadtverwaltung auf Zuschüsse aus der Investitionsbank zur Behebung der Arbeitslosigkeit abgelehnt wurden" 54 .

Einzelne Kontrollberichte zeigen die begrenzte Durchschlagskraft der ZusatzInvestitionspläne auf dem Arbeitsmarkt. So waren beispielsweise im Land Sachsen während des zweiten Quartals 1949 insgesamt 222377 Personen durch die Arbeitsämter vermittelt worden, darunter 96117 Frauen $^{55}$. Am 25. Juli wurden 27960 Personen gezählt, die aufgrund der Sofortprogramme einen Arbeitsplatz erhalten hatten. Im Vergleich zur geforderten Größenordnung (33910) betrug der

53 BAB, DC 15/60, Bl. $1 \mathrm{f}$.

54 LA Magdeburg LHA, Rep. K MW, Nr. 10076, Bl. 60, Amt für Arbeit und Sozialfürsorge Zeitz am 7. 7. 1949 an den Minister für Arbeit und Sozialpolitik von Sachsen-Anhalt.

55 BAB, DC 15/60, Bl. 5-7, hier Bl. 5, Kontrollbericht der HA Wirtschaftsplanung (Abt. Plankontrolle) der sächsischen Landesregierung vom 10. 8. 1949. 
Erfüllungsstand zwar 82,3 Prozent. Angesichts der Gesamtvermittlungen durch die Arbeitsämter stellte dies jedoch nur einen bescheidenen Beitrag zur Entlastung auf dem Arbeitsmarkt dar, auch wenn die unterschiedlichen Erhebungszeiträume berücksichtigt werden müssen (Zeitraum versus Stichtag). Die zahlenmäßig größte Gruppe der laut Zusatzplan am 25. Juli beschäftigten Personen war zu Enttrümmerungsarbeiten eingesetzt worden (16710). Es folgten: 4556 Arbeitskräfte für Meliorationsarbeiten, 4000 in der Forstwirtschaft und 2403 für Straßenbautätigkeiten. Vergleichsweise unbedeutend blieben Arbeitsprojekte beim Wasserstraßenbau (124 Personen) und bei der Neubauernhilfe (167). Abschließend sprachen sich die Kontrolleure dafür aus, den einzelnen Investitionsträgern größere Spielräume bei der Vergabe der ihnen zugeteilten Finanzmittel zu gewähren, „um so den jeweiligen Forderungen der Praxis gerecht zu werden" 56 . Einen kritischen Eindruck vermittelte auch der Kontrollbericht der Hauptabteilung Wirtschaftsplanung bei der Landesregierung von Sachsen-Anhalt ${ }^{57}$. In den Monaten April bis August 1949 waren hier 12408 Personen vermittelt worden; die einzelnen Objekte des Zusatzplanes beschäftigten am 31. Juli insgesamt 16729 Arbeitskräfte. Der Schwerpunkt lag in Sachsen-Anhalt beim Straßenbau (7598), gefolgt vom Wasserstraßenbau (2993), der Forstwirtschaft (2933), Enttrümmerungsarbeiten (2799) und sonstigen Projekten (163). Im Zuge der Realisierung des Zusatzplanes hatten sich Schwierigkeiten ergeben, die auf die späte Veröffentlichung des Zusatzplanes, vor allem aber auf ausbleibende Materiallieferungen zurückgeführt wurden, welche die Produktion in den jeweiligen Betrieben fast zum Erliegen gebracht hatten. Bei der Analyse einzelner Kreise stellten die Berichterstatter fest, "daß von einer Planung, vom Standpunkt des Volkswirtschaftsplanes gesehen, nur sehr schwache Anfänge zu bemerken sind “58. Es fehle noch eine „zusammenfassende Dienststelle" zur Koordinierung der Verwaltungstätigkeit mit dem Ziel, eine rasche und effiziente Durchführung des Zusatzplanes zu erreichen.

Sowohl die HVAS als auch die Hauptverwaltung Wirtschaftsplanung ließen die Landesregierungen über die Fortsetzung des Zusatz-Investitionsplanes im Ungewissen. Diese fragten zu Beginn des Jahres 1950 beim mittlerweile neu gebildeten Ministerium für Arbeit und Gesundheitswesen in Berlin an, „ob für 1950 ähnliche Maßnahmen durchgeführt und entsprechende Mittel zur Verfügung gestellt werden müssen" 59 . $\mathrm{Da}$ zu diesem Zeitpunkt die bereitgestellten Mittel noch nicht vollständig ausgegeben waren, rechneten einzelne Landesministerien mit einem Ende der beschäftigungspolitischen Programme erst zum Frühjahrsbeginn. Grundsätzliche Überlegungen und die Ausarbeitung von alternativen Konzepten waren allerdings unumgänglich, wollte man nicht ein automatisches Ansteigen der Arbeitslosenzahlen in Kauf nehmen. Die sächsische Hauptabteilung Wirtschaftsplanung setzte sich daraufhin mit dem Ministerium für Planung der DDR in Ver-

56 Ebenda, Bl. 7.

57 BAB, DC 15/60, Bl. 9-11, Kontrollbericht vom 1. 9. 1949.

58 Ebenda, Bl. 14.

$59 \mathrm{BAB}, \mathrm{DQ} 2 / 1729$, Ministerium für Arbeit und Gesundheitswesen von Sachsen-Anhalt am 5.1. 1950 an das Ministerium für Arbeit und Gesundheitswesen. 
bindung und beantragte weitere Finanzmittel für Beschäftigungsvorhaben ${ }^{60}$. Letztlich kam es allerdings nicht zur Aufstellung eines neuen Sofortprogramms. Planung und Lenkung der Arbeitskräfte wurden vielmehr Bestandteile der Volkswirtschaftspläne; wie bereits angedeutet, überlagerte dies die bisherige arbeitsmarktpolitische Debatte. Da die Zahl der offenen Stellen nicht sehr groß und die allgemein angespannte Haushaltlage eigene arbeitsmarktpolitische Initiativen nicht zuließ, schien ein Ansteigen der Arbeitslosenzahlen unausweichlich zu sein. Das sächsische Ministerium für Arbeit und Gesundheitswesen berichtete, daß im Laufe des Monats Januar 1950 die Erwerbslosenzahl von 102000 auf 124322 angestiegen sei ${ }^{61}$. Eine Statistik des Ministeriums für Arbeit und Gesundheitswesen in Berlin ergab, daß der Höhepunkt des Zusatz-Investitionsplanes bereits im November 1949 überschritten worden war62: Die Zahl der beschäftigten Personen war zunächst von 48882 (Juni) auf 81893 (Juli) gestiegen. Nach einem Einbruch im August 1949, als nur 63222 Arbeitskräfte gezählt wurden, stabilisierte sich die Zahl bei knapp über 80000 . Im Oktober war der Höchststand erreicht worden (84 425). Die Zahl der im Zuge des Beschäftigungsprogramms eingestellten Arbeiter sank auf 51255 (Dezember). Somit war das Ende des Sofortprogramms bzw. des Zusatz-Investitionsplanes absehbar.

\section{Lobnpolitik}

Im folgenden soll keine Gesamtdarstellung der staatlichen Lohnpolitik zwischen Kriegsende und DDR-Gründung angestrebt werden, vielmehr geht es darum, der Frage nachzugehen, inwieweit die Gestaltung der Löhne und Gehälter von seiten der staatlichen Zentralverwaltungen gezielt zur Steuerung des Arbeitskräftepotentials eingesetzt wurde. Mit dem Ende des Jahres 1945 verhängten Lohnstopp hatte die SMAD frühzeitig den Tarifparteien die Möglichkeit genommen, die Höhe von Löhnen und Gehältern im Rahmen von Verhandlungen auszuhandeln ${ }^{63}$. Die deutsche Arbeitsverwaltung erhielt den Auftrag, über die Einhaltung dieser Bestimmungen zu wachen. Ein wichtiges Regulativ zur Steuerung des Arbeitsmarktes war damit faktisch beseitigt worden. Diese Regelung trafen aber auch die drei westlichen Siegermächte zeitweise für ihr Besatzungsgebiet: Hier wurde der Lohnstopp im November 1948 wieder aufgehoben ${ }^{64}$. Für die SBZ galt: Lohn- und Gehaltserhöhungen gingen im wesentlichen auf Initiativen der sowjetischen Militäradministration zurück, wobei sich hierbei die sowjetischen Reparationsinteressen indirekt bemerkbar machten. Dies zeigte sich etwa beim Befehl Nr. 234 und der damit verbundenen Verbesserung der Versorgung von Wismut-

60 $\mathrm{BAB}, \mathrm{DQ} 2 / 1755$, Ministerium für Arbeit und Gesundheitswesen von Sachsen am 19.1. 1950 an das Ministerium für Arbeit und Gesundheitswesen, S. 1.

61 BAB, DQ 2/1729, Ministerium für Arbeit und Gesundheitswesen der Landesregierung Sachsen am 2. 2. 1950 an das Ministerium für Arbeit und Gesundheitswesen.

62 BAB, DQ 2/1755, Aktenvermerk von Oberreferent Grodecki vom 16. 2. 1950.

63 SMAD-Befehl Nr. 180 vom 22. 12.1945 und die Richtlinien, welche die ZVAS dazu herausgab, in: Arbeit und Sozialfürsorge 1 (1946), S. 12 f. Bereits mit der Anordnung Nr. 1 vom 23. 7. 1945 hatte die SMAD verfügt, daß „die unmittelbar vor Beendigung der Kampfhandlungen gültigen Lohnund Gehaltssätze bestehen bleiben. “ Dazu den Beitrag von Gustav Schaum, Preise und Löhne, in: ebenda, S. $84 \mathrm{f}$.

64 Akten zur Vorgeschichte der Bundesrepublik, Bd. 4, S. 803, Anm. 14. 
Arbeitern. Darüber hinaus besaßen der Bergbau, später dann auch weitere Branchen der Grundstoff- und Schwerindustrie, oberste Priorität für die SMAD. In diesen Sektoren erhielten die Arbeiter bald deutliche Lohnzuwächse. Vorrangiges Ziel der Lohnpolitik war jedoch zuallererst die Steigerung der Arbeitsproduktivität in den Betrieben; dagegen spielten Überlegungen zur Steuerung der erwerbstätigen Bevölkerung in bestimmten Wirtschaftszweigen eine eher untergeordnete Bedeutung.

Die sowjetische Besatzungsmacht sowie die deutschen Landesverwaltungen befürchteten ein teilweise rasantes Ansteigen der Löhne, das dann automatisch Auswirkungen auf die Preisentwicklung gehabt hätte. Die Angst vor Inflation war somit ein wesentlicher Grund dafür, daß der Lohnstopp verhängt wurde. Das thüringische Landesamt für Arbeit und Sozialfürsorge berichtete der ZVAS Ende 1945 von "wilde[n] Lohnbewegungen“, die offenbar nicht nur vereinzelt auftraten, sondern aus „allen Teilen“ des Landes gemeldet wurden ${ }^{65}$. Zahlreiche Betriebe hielten sich offenbar nicht an den verhängten Lohnstopp. So ergab eine vom Landesarbeitsamt Sachsen am 5. Januar 1946 in Auftrag gegebene Erhebung, daß von insgesamt 9638 erfaßten Betrieben mit mehr als 20 Belegschaftsmitgliedern 1780 eigenmächtig Lohnerhöhungen, mitunter auch Lohnkürzungen vorgenommen hatten ${ }^{66}$. Das bedeutete, daß sich rund 18,5 Prozent der registrierten Betriebe über den SMAD-Befehl Nr. 180 hinweggesetzt hatten und die Lohnpolitik als Bestandteil unternehmerischen Handlungsspielraumes ansahen. Allerdings signalisierte die Landesverwaltung in den Fällen ihre Zustimmung, bei denen die Lohnsumme insgesamt nicht verändert wurde, sondern nur eine Nivellierung der Löhne und Gehälter erfolgt war. Darüber hinaus hatten sich Lohnerhöhungen durch den Übergang von der Akkord- auf die Zeitlohnarbeit unter Beibehaltung des Akkordrichtlohnes ergeben. Auch der Befehl über "gleiche Entlohnung für gleiche Arbeit" 67 zeitigte Folgen und führte, wenn auch stark begrenzt, zu einer Erhöhung der Löhne für Frauen und Jugendliche. Eine Rückführung der Löhne und Gehälter auf das ursprüngliche Niveau strebte die Arbeitsverwaltung jedoch nicht an, da sie Unruhen unter den Belegschaften befürchtete ${ }^{68}$.

Die SMAD bestand auf der strikten Einhaltung des Lohnstopps bzw. behielt sich ein Vetorecht bei der Gestaltung der Löhne durch die DVAS vor. In dem Zusammenhang wurden zuvor erlassene Befehle sogar neu interpretiert. So wies etwa der SMAD-Vertreter Prof. Formin darauf hin, daß der Befehl Nr. 253 von deutschen Stellen zu Lohnerhöhungen genutzt und damit falsch ausgelegt worden sei69. Lohnpolitik war nicht nur eine Angelegenheit von sowjetischer Besatzungsmacht und deutscher Arbeitsverwaltung: Auch hier machte sich die bis ungefähr Mitte 1946 relativ starke Position der Landesverwaltungen sowie der

65 BAB, DQ 2/3923, Bl. 42, Protokoll über Besprechung der ZVAS mit Vertretern des Landesamtes für Arbeit und Sozialfürsorge in Weimar am 18. 12. 1945. Entsprechende Meldungen aus Brandenburg gab es noch im Sommer 1947. BLHA, Ld. Br. Rep. 206, Bd. 3051, Aktennotiz der Abt. Arbeitsrecht, Arbeit und Löhne vom 29. 7. 1947 für Ministerialdirektor Fischer betr. Befehl Nr. 180.

$66 \mathrm{BAB}, \mathrm{DQ} 2 / 797$, Notiz der DVAS (o.D.).

67 Vgl. SMAD-Befehl Nr. 253 vom 17. 8. 1946, in: Arbeit und Sozialfürsorge 1 (1946), S. 306.

$68 \mathrm{BAB}, \mathrm{DQ} 2 / 797$, Notiz der DVAS (o.D.).

$69 \mathrm{BAB}, \mathrm{DQ} 2 / 2076$, Niederschrift der DVAS-Abt. I b über Besprechung mit SMAD am 17. 7. 1947, S. 3 . 
Kommunalverwaltungen trotz der finanzpolitisch engen Handlungsspielräume bemerkbar. Im Zuge der Kompetenzverlagerung von den Landesverwaltungen zu den Zentralverwaltungen erfolgte eine immer stärkere Absprache zwischen DVAS und Deutscher Zentralfinanzverwaltung 70 . Dennoch kam es immer wieder vor, daß die SMAD „Aufträge oft sehr kurzfristig erteilt[e]“, so daß sich die DVAS gezwungen sah, in diesen Fällen ohne Rücksprache mit der Zentralfinanzverwaltung zu handeln ${ }^{71}$.

Bei der Ausarbeitung des Zweijahrplanes verstärkte sich die Bedeutung der betrieblichen Produktivitätssteigerung weiter. Auch die Lohnpolitik wurde diesem Ziel immer mehr untergeordnet, wie die Diskussion über die Leistungslöhne anschaulich zeigt ${ }^{72}$. Karlshorst unterstützte diese Politik nicht nur, sondern trieb die Entwicklung selber aktiv voran: Die deutsche Arbeitsverwaltung erhielt wiederholt den Auftrag, entsprechende Richtlinien auszuarbeiten und vorzulegen ${ }^{73}$. Dabei ergaben sich rasch Unstimmigkeiten zwischen der DWK und einzelnen Hauptverwaltungen. So kritisierte etwa der stellvertretende DWK-Vorsitzende Fritz Selbmann, daß die HVAS keine unterschiedliche Behandlung von volkseigenen und privaten Betrieben vorgenommen habe. Diese Tendenz sei nicht zufällig, sondern eine "ausgesprochen opportunistische Auffassung, die den neuen Charakter unserer Wirtschaft bewußt ignoriert und Arbeitspolitik im alten reformistischen Sinne zu machen versucht" 74 . Dahinter standen allerdings auch Versuche, der Arbeitsverwaltung das Gebiet der Lohnpolitik streitig zu machen. Selbmann warf der HVAS vor, daß sie bestrebt sei, „die Mitarbeit der Fachverwaltungen der DWK-Industrie auszuschalten." Er machte dafür „bürokratische[] Tendenzen“ innerhalb der HVAS verantwortlich und rief sogar „zur größten Wachsamkeit gegenüber allen Vorschlägen und Entwürfen" von seiten dieser Verwaltung auf75. Im einzelnen plädierte Selbmann dafür, daß die DWK-Hauptverwaltungen sowie die Industriegewerkschaften nur Rahmenrichtlinien herausgeben und der Übergang zum progressiven Leistungslohn ,auch ohne jede Einschaltung bürokratischer Instanzen" zu erfolgen hat ${ }^{76}$. Im Frühjahr 1949 arbeiteten nach Angaben der HVAS rund 40 Prozent der in volkseigenen Betrieben Beschäftigten im Leistungslohnbereich. Bei den SAG-Betrieben kann der Anteil nicht genau angegeben werden: Er schwankte in der metallverarbeitenden Industrie zwischen 40 und 60 Prozent, im Bergbau zwischen 20 und 35 Prozent, und in der chemischen Industrie lag er zwischen 20 und 40 Prozent. Auch bei den Ländern gab es erhebliche Differenzen. Spitzenreiter war offenbar Sachsen-Anhalt, wo 43,3 Prozent der VEB-Beschäftig-

70 BAB, DQ 2/3846, DVAS-Vizepräsident Herm am 7. 1. 1948 an die Deutsche Zentralfinanzverwaltung. Vgl. Zschaler, Die Entwicklung einer zentralen Finanzverwaltung.

$71 \mathrm{BAB}, \mathrm{DQ} 2$ 3846, DVAS-Vizepräsident Herm am 7. 1. 1948 an die Deutsche Zentralfinanzverwaltung.

72 Vgl. zur Propagierung der Leistungslöhne Hübner, Konsens, Konflikt und Kompromiß, S. 27-36.

$73 \mathrm{BAB}, \mathrm{DQ} 2 / 1535, \mathrm{Bl}$. 114, Aktennotiz der HVAS-Abt. III über eine Besprechung mit Morenow in Karlshorst am 20.8. 1948.

74 SAPMO, NY 4182/951, Bl. 275-280, hier Bl. 280, Stellungnahme der DWK (Sekretariat Industrie) vom 16. 9. $1948 \mathrm{zu}$ den Richtlinien über die Lohngestaltung in den Betrieben der SBZ mit Anschreiben Selbmanns an Walter Ulbricht.

75 Ebenda, Bl. 276.

76 Ebenda, Bl. 278. 
ten in den Leistungslohnsektor fielen; in Thüringen waren es 33 Prozent, in Sachsen 27 Prozent und in Brandenburg nur 17 Prozent $^{77}$.

\section{Wohnungsbaupolitik}

Auch der Wohnungsbau unterlag in der SBZ keineswegs nur den Anforderungen der Arbeitskräftelenkung, sondern war vielmehr von Ad-hoc-Maßnahmen geprägt, die zudem sehr stark von den Landes- bzw. Kommunalverwaltungen sowie vor allem den Besatzungsmächten mit entschieden wurden. Die Notlage angesichts der umfangreichen Zerstörung von Wohnraum während des Zweiten Weltkrieges galt im übrigen für alle vier Besatzungszonen ${ }^{78}$. Insofern war es wenig überraschend, als die vier Siegermächte im Alliierten Kontrollrat am 8. März 1946 ein Wohnungsgesetz beschlossen ${ }^{79}$, das die Verteilung des knappen Gutes auch der staatlichen $Z$ wangsbewirtschaftung unterwarf.

Frühzeitig beklagte sich die Arbeitsverwaltung über die unzureichende Wohnraumversorgung und machte darauf aufmerksam, daß diese Mangelsituation direkte Auswirkungen auf die Arbeitskräftelenkung hatte. Darüber hinaus waren zahlreiche Arbeiter nicht bereit, den Arbeitsplatz unter Inkaufnahme der Trennung von der Familie zu wechseln. Anläßlich einer gemeinsamen Besprechung von DVAS und einigen wirtschaftlichen Zentralverwaltungen wurde allgemein beklagt: „Die Werke sind nicht einmal in der Lage, die angeforderte Zahl an Arbeitskräften ohne Angehörige unterzubringen, und wenn dies möglich wäre, so ergibt sich eine weitere Schwierigkeit, nämlich, daß sich die Arbeitskräfte nicht von der Familie trennen wollen." 80 Um die Erfassung und Vergabe von Wohnraum einheitlich für alle Länder der SBZ zu regeln, erließ die DVAS zunächst einmal am 27. Juli 1946 eine Durchführungsverordnung. Da diese auch die gewerblichen Räume erfaßte, kam es offensichtlich zu einer Kontroverse zwischen der Berliner Zentralverwaltung und einzelnen Landesverwaltungen, die sich dagegen ausgesprochen hatten. Die DVAS beharrte jedoch auf ihrer Position und wies explizit darauf hin, daß entsprechende Verordnungen der Länder Thüringen und Sachsen nichtig seien ${ }^{81}$.

Die einzelnen wohnungsbaupolitischen Maßnahmen hingen in den ersten Nachkriegsjahren mitunter sehr stark von den jeweiligen Arbeitskräfteanforderungen ab. Dabei sollten die Arbeiter der Grundstoff- und Schwerindustrie zuerst mit Wohnraum versorgt werden, insbesondere die Beschäftigten der Wismut AG. Der rasant angestiegene Arbeitskräftebedarf hatte dort, darauf ist bereits hingewiesen worden, zu einer Zuspitzung der ohnehin angespannten Wohnungssituation geführt. Da der Neubau von Wohnraum nicht so schnell abgeschlossen werden konnte - dieser wurde anfangs erheblich durch den Mangel an Baumate-

77 SAPMO, DY 34/4187, Auszug aus einer Besprechung mit Morenow in Karlshorst am 22. 4. 1949, S. 1.

78 Vgl. Führer, Mieter, Hausbesitzer, Staat und Wohnungsmarkt; Schildt, Wohnungspolitik; Schulz, Wiederaufbau in Deutschland; Wagner, Sozialstaat gegen Wohnungsnot.

79 Gesetz Nr. 18, in: Amtsblatt des Kontrollrats in Deutschland, S. 117-121.

so BAB, DO 2/30, Bl. 114f., hier Bl. 114, Bericht Görlichs über die Sitzung am 7. 5. 1946.

81 SAPMO, NY 4182/951, Bl. 268f., Jenny Matern am 16. 3.1948 an das Ministerium für Arbeit und Sozialfürsorge des Landes Sachsen-Anhalt. 
rialien behindert -, griff die Arbeitsverwaltung in der Not zu anderen Lösungen: der „Umsiedlung“ von Wohnungsinhabern, die nicht im Uranbergbau bzw. Steinkohlenbergbau tätig waren. Aus Sicht der Behörden stellten die Flüchtlinge und Vertriebenen eine soziale Gruppe dar, die nicht ortsgebunden war und insofern als erste für diese Aktion prädestiniert schien. Gleichzeitig unterstrich die HVAS des öfteren gegenüber den Landesverwaltungen, daß die „Umsiedlungsmaßnahmen [...] auf freiwilliger Grundlage" erfolgen sollten ${ }^{82}$. Der erhoffte Erfolg blieb allerdings aus, da die Länder nicht in der Lage waren, ihrerseits zusätzlichen Wohnraum für die eintreffenden „Umsiedler“ in größerem Umfange zur Verfügung zu stellen. Das Ministerium des Innern der Landesregierung Sachsen-Anhalt faßte den bisherigen Verlauf kurz zusammen: „Von Einzelfällen abgesehen, wird die Umsetzungsaktion auf der bisherigen Basis keine größeren Erfolge aufzeigen." ${ }^{83}$ Die HVAS blieb trotzdem bei ihrer Linie und sprach sich auch weiterhin gegen eine gesetzliche Regelung der Binnenumsiedlung aus, „da diese Maßnahmen das verfassungsmäßig gewährleistete Recht der Freizügigkeit in vollem Umfange außer Kraft setzen würden"84. Das bedeutete letztlich, daß sogar im Erzgebirge mit den Uranbergwerken, die bei der Gewinnung von Arbeitskräften höchste Priorität genossen, das Wohnungsproblem erst zu dem Zeitpunkt einer Lösung näher kommen konnte, als der Arbeitskräftebedarf bei der Wismut AG insgesamt deutlich gesunken war.

Bei einer Bilanzierung der Wohnungsbaupolitik kam die HVAS zu einem sehr ernüchternden Ergebnis. Allem Anschein nach war es nicht gelungen, eine Umverteilung des verfügbaren Wohnraumes nach scheinbar egalitären Prinzipien zu verwirklichen. So kamen denn auch die Mitarbeiter der Berliner DWK-Hauptverwaltung zum Ergebnis, daß die "ehemaligen aktiven Nazis und Militaristen, Kriegsverbrecher, Junker sowie auch Fabrikanten und Großhändler [...] viel besser mit Wohnraum versorgt [sind] als die werktätige Bevölkerung, besonders als die Aktivisten der volkseigenen Betriebe und die Antifaschisten." 85 Entscheidend für die Arbeitskräftelenkung war die Tatsache, daß der zur Verfügung gestellte Wohnraum oftmals für andere Zwecke verwendet wurde: In Sachsen waren dies bis zum Oktober 1946 immerhin 1615000 qm, im Oktober 1948 sogar 2396000 qm Wohnfläche; Mecklenburg-Vorpommern meldete im Oktober 1946365000 qm, zwei Jahre später schon 852000 qm$^{86}$. Der Bericht gibt keine Auskunft über die weitere Verwendung des Wohnraumes. Insgesamt bleibt aber festzuhalten, daß durch diese Fehlallokation der bestehende Wohnraummangel weiter verschärft wurde und Versuche des zwischen- und überbezirklichen Ausgleichs von Arbeitskräften zum Scheitern verurteilt waren bzw. in den Anfängen stecken blieben. Auf Anregung der HVAS erteilte der DWK-Vorsitzende Heinrich Rau den zuständigen Landesministerien auf einer gemeinsamen Konferenz den Auftrag,

$82 \mathrm{BAB}, \mathrm{DO} 2 / 58, \mathrm{Bl}$. 63, HVAS (Donau) am 14. 3. 1949 an das Ministerium für Wirtschaft und Verkehr der Landesregierung Sachsen-Anhalt.

83 Ebenda, Bl. 83, MdI (Abt. Bevölkerungspolitik) der Landesregierung Sachsen-Anhalt am 11.7. 1949 an die DVdI (HA Umsiedler).

84 Ebenda, Bl. 86, HVAS (Litke) an die DVdI.

${ }_{85}$ SAPMO, NY 4090/561, Bl. 50-53, hier Bl. 50, Denkschrift (o. Verf.) vom 18. 7. 1949 zur Wohnungsbaupolitik in der SBZ.

86 Ebenda, Bl. 51. 
eine Sonderaktion zur Erfassung von Wohnraum durchzuführen ${ }^{87}$. Hauptadressaten bei der Wohnungsvergabe sollten „Umsiedler" sowie generell Erwerbstätige sein. Das endgültige Ergebnis der statistischen Erhebung mußte aus Sicht der Arbeitsverwaltung eher bescheiden wirken. So wurden in allen fünf Ländern der SBZ insgesamt 6372 Wohnungen und 99608 Zimmer (mit jeweils 13 qm) gezählt, die neu belegt werden konnten ${ }^{88}$. Dieser Neubestand reichte jedoch bei weitem nicht aus - weder zur Versorgung der Flüchtlinge und Vertriebenen noch zur Neuansiedlung von Arbeitskräften in Großstädten bzw. bestehenden oder geplanten industriellen Schwerpunktgebieten.

\section{Arbeitskräftelenkung als Bestandteil der allgemeinen Wirtschaftsplanung}

Während die zuvor beschriebenen Instrumente direkte Auswirkungen auf die Fluktuation von Arbeitskräften haben konnten und mitunter schon die Anreizfunktion erkennen ließen, trifft dies für die allgemeine Wirtschaftsplanung in dieser Frühphase nicht gleichermaßen zu. Der Übergang von der kriegsbedingten Zwangsbewirtschaftung, die sehr stark den wirtschaftlichen Folgen des Krieges und den Anforderungen der Besatzungsmächte geschuldet war, hin zur Planwirtschaft bzw. Zentralverwaltungswirtschaft zog sich über einen längeren Zeitraum hin. Das langfristige Ziel stand dabei für die SED- und DWK-Führung fest: In enger Anlehnung an das sowjetische Vorbild sollte der Einsatz der Produktionsfaktoren über mehrere Jahre festgelegt werden. Konjunkturelle Schwankungen des Wirtschaftsablaufes, die in den zwanziger und noch Anfang der dreißiger Jahre für die westeuropäischen Staaten beherrschend und damit auch für die deutschen Kommunisten in ihren ökonomischen Überlegungen prägend gewesen waren, schienen der Vergangenheit anzugehören - so jedenfalls die Prognosen und Erwartungen der SED-Führung. Die Rahmenbedingungen für diese grundlegende Neuordnung der Wirtschaft in der SBZ wurden mit wesentlicher Unterstützung der SMAD frühzeitig festgesetzt: Dazu zählten vor allem die Bodenreform, die Sequestrierung von Großunternehmen und Betrieben sowie die Beschlagnahmung von Bankguthaben. Anschließend sollten dann zentrale Wirtschaftspläne zunächst für die Länder, dann aber auch für die gesamte SBZ erstellt werden. In dem Zusammenhang mußte schließlich das verfügbare Arbeitskräftepotential auf die einzelnen Wirtschaftssektoren bzw. -zweige verteilt werden. Wie erfolgte nun die Planung zur Verteilung der Arbeitskräfte in der SBZ? Welche Antwort fand die SED-Führung auf das grundsätzliche Problem der Zuteilung knapper Güter, das eben auch für dieses Wirtschaftssystem galt?

In der unmittelbaren Nachkriegszeit wurden Konzepte über eine planvolle Lenkung und Steuerung des Arbeitskräftepotentials zunächst nur von der ZVAS/ DVAS ausgearbeitet. Dagegen äußerten sich die beiden Arbeiterparteien SPD und KPD bei ihren ersten programmatischen Richtlinien zur Umgestaltung der Wirtschaft sehr zurückhaltend und unverbindlich zu einzelnen arbeitsmarktpoliti-

87 SAPMO, NY 4090/561, Bl. 60, HVAS (Litke) am 15. 9. 1949 an Otto Grotewohl.

88 Ebenda, Bl. 56. Der statistischen Übersicht ist ein ausführlicher Bericht der HVAS für die SMADAbt. Arbeitskraft vom 1. 9. 1949 beigefügt. Vgl. cbenda, Bl. $54 \mathrm{f}$. 
schen Instrumenten und Programmen ${ }^{89}$. Dies lag unter anderem an der Tatsache, daß die Arbeitsverwaltung aufgrund ihrer eigentlichen Aufgabenstellung sehr viel stärker unter dem Erwartungsdruck stand, den im Zuge des Zweiten Weltkrieges aufgetretenen demographischen Verwerfungen der Erwerbsbevölkerung einigermaßen zu begegnen und auf die damit zusammenhängenden Problemlagen Antworten zu finden. Im Mittelpunkt des Interesses stand für die Arbeitsverwaltung zunächst der Verwaltungsaufbau: In einem Rundschreiben vom 4. Januar 1946 wies die ZVAS ausdrücklich darauf hin, daß „eine planvolle Gestaltung des Arbeitseinsatzes“ nur erreicht werden könne, „wenn die Durchführung aller Maßnahmen des Arbeitseinsatzes durch eine Stelle erfolgt ${ }^{\prime \prime 90}$. Damit verband sie konkret die Forderung nach einer einheitlichen Verwaltungsstruktur, die sich allerdings nicht so rasch verwirklichen ließ ${ }^{91}$.

Mit dem ständig wachsenden Arbeitskräftebedarf traten ungefähr im Sommer 1946 die Planungsarbeiten der DVAS in eine neue Phase ein. Die SED-Führung verlangte die Bereitstellung von 162000 Arbeitern, „um die elementarsten Bedürfnisse der Wirtschaft zu befriedigen", wie der DVAS-Präsident in einer Aktennotiz vermerkte ${ }^{92}$. Akuter Arbeitskräftemangel bestand zu diesem Zeitpunkt vor allem in der Textilindustrie, der Grundstoff- und Schwerindustrie (Bergbau, Maschinenbau, Metallindustrie und Chemie) sowie bei der Oderregulierung. Die SEDFührung stellte insgesamt vier Forderungen auf: So sollte die Umschulung von ungelernten Arbeitern vorangetrieben, in den Betrieben Männer durch Frauen und Körperbehinderte ersetzt sowie die Schulentlassenen und Jugendlichen mit Arbeit versorgt werden ${ }^{93}$. Dahinter standen im wesentlichen zwei Ziele, die eng miteinander verwoben waren. Es ging zum einen um eine erhebliche Vergrößerung der Erwerbstätigenzahlen durch die Eingliederung von Bevölkerungsgruppen, die bisher außerhalb des Arbeitslebens standen. Zum anderen sollten männliche Arbeiter freigesetzt werden, um den Arbeitskräftebedarf in der Schwerindustrie befriedigen zu können. Die enge Verzahnung der Planung des Arbeitskräfteeinsatzes mit Fragen der Berufsberatung, -ausbildung und -umschulung war daher naheliegend: Die Abteilung II (Erfassung und Arbeitslenkung der Bevölkerung) bei der Berliner Zentralverwaltung legte am 31. August 1946 eine Denkschrift vor, in der die Verknüpfung der einzelnen arbeitsmarktpolitischen Gebiete angestrebt wurde ${ }^{94}$.

89 Vgl. SAPMO, DY 28/II 2/3, Bl. 14, Richtlinien der Wirtschaftspolitik, beschlossen vom ZA der SPD am 21. 9. 1945; SAPMO, RY 1/I 2/2/22, Bl. 47-51, Richtlinien der KPD zur Wirtschaftspolitik [29. 12. 1945]. In einem Entwurf für eine wirtschaftspolitische Denkschrift vom 26.1. 1946 verwies die KPD explizit auf die Forderung von seiten der SMAD an die deutschen Zentralverwaltungen, einen Wirtschaftsplan für 1946, gegliedert in Vierteljahrespläne, auszuarbeiten. Nach Angaben der KPD bestand ein wesentliches Ziel in der „rationelle[n] Verwendung der zur Verfügung stehenden Arbeitskräfte und deren Lenkung an Stellen des dringenden Bedarfs." SAPMO, NY 4182/951, Bl. 84-101, hicr Bl. 86. Gleichzeitig betonte die KPD das Recht und die Verpflichtung jedes Erwerbsfähigen zu arbeiten. Ebenda, Bl. 90.

96 BAB, DQ 2/2066, Rundschreiben Nr. 40 der ZVAS vom 4. 1. 1946, S. 1. Das Rundschreiben wurde an die Landesarbeitsämter verschickt. Ein erster Entwurf der ZVAS-Abt. II stammte vom 14. 12. 1945 und befindet sich in: BAB, DQ 2/2064.

91 Vgl. S. 21-43.

$92 \mathrm{BAB}, \mathrm{DQ} 2 / 142$, Bl. 1-4, hier Bl. 1, Aktennotiz von Gustav Brack vom 29. 7. 1946.

93 Ebenda, Bl. 2.

94 $\mathrm{BAB}, \mathrm{DQ} 2 / 2064$. 
Anschließend versuchte die DVAS Absprachen mit den übrigen Zentralverwaltungen herbeizuführen, um den jeweiligen Arbeitskräftebedarf einheitlich erfassen zu können. Im Zuge der langsam beginnenden allgemeinen Wirtschaftsplanung sollte somit bereits Ende 1946 auch eine Arbeitskräfteplanung erfolgen"5. Zusammen mit der Zentralverwaltung der Industrie führte die DVAS eine grundsätzliche Aussprache, in deren Verlauf zunächst nur die Schwierigkeiten und Hindernisse auf diesem Weg erörtert werden konnten. Da die Anforderungen der sowjetischen Besatzungsmacht und vor allem der Bedarf für die SAG-Betriebe zu diesem Zeitpunkt noch nicht abgesehen werden konnten, waren der DVAS noch enge Handlungsspielräume gesetzt. Anfang 1947 erstellten zunächst die Landesbzw. Provinzialverwaltungen erste Produktionspläne für das laufende Jahr, in denen der Arbeitskräftebedarf konkret angegeben wurde, der mehrheitlich auf den sowjetischen Befehlen für die einzelnen Reparationsaufgaben beruhte ${ }^{96}$. Der DVAS gelang es nur allmählich, von der Zentralverwaltung für Industrie Zahlenangaben für den Arbeitskräftebedarf zu erhalten ${ }^{97}$. Eine bessere Absprache zwischen den Zentralverwaltungen wurde erst erreicht, als mit der DWK-Hauptverwaltung Wirtschaftsplanung eine zentrale Instanz geschaffen worden war ${ }^{98}$. Darüber hinaus schaltete sich die SMAD in zunehmenden Maße in die Planungsarbeiten ein ${ }^{99}$. Auch der Einfluß der SED-Führung wurde immer stärker: Im Zuge der Ausarbeitung des Zweijahrplanes, der innerhalb des Zentralsekretariats zum Teil kontrovers diskutiert wurde ${ }^{100}$, legte die Abteilung Arbeit und Sozialfürsorge beim SED-Zentralsekretariat Vorschläge für die Nachwuchslenkung vor ${ }^{101}$; das Frauensekretariat arbeitete einen Arbeitsplan „über die Mitarbeit der Frauen bei der Durchführung der Wirtschaftspläne“ aus ${ }^{102}$. Schwierigkeiten ergaben sich jedoch zwischen der DWK-Hauptverwaltung Wirtschaftsplanung und einzelnen industriellen Hauptverwaltungen, die ihre Ressortinteressen durchzusetzen versuchten: So monierte Bruno Leuschner, daß nach wie vor ,jeder Industriezweig vom Standpunkt seiner eigenen Interessen aus plant und die Pläne dann mechanisch koordiniert werden“. Sollte sich dieses „alte Prinzip“ erneut durchsetzen,

${ }^{95} \mathrm{BAB}, \mathrm{DQ} 2 / 1963$, Aktenvermerk der DVAS-Abt. I vom 19. 12. 1946.

${ }_{96}$ BAB, DQ 2/1963, Produktionsplan des Landesarbeitsamtes Sachsen vom 29.11. 1946; ebenda, Minister für Arbeit und Sozialpolitik der Provinzialregierung Sachsen-Anhalt am 7.1. 1947 an die DVAS; ebenda, Ministerium für Wirtschaft, Arbeit und Verkehr des Landes Thüringen am 22. 2. 1947 an die DVAS.

97 BAB, DQ 2/1963, Aktenvermerk der DVAS-Abr. I b vom 12. 3. 1947.

${ }^{98}$ Pläne zur Gründung eines „Deutschen Amtes für Wirtschaftsplanung“ gab es innerhalb der SEDFührung offensichtlich schon im Herbst 1946. Der Strukturplan sah unter anderem eine Hauptabteilung Arbeitskräfte vor. SAPMO, NY 4036/734, Bl. 221. Diese Überlegungen waren sehr wahrscheinlich mit Karlshorst zuvor abgesprochen worden. Die SMAD-Führung erhielt am 26.10. 1946 eine überarbeitete Version des Strukturplanes, der nicht nur die Zentralebene erfaßte, sondern auch die Landes-, Bezirks- und Kreisebene mit einschloß. SAPMO, NY 4182/1192, Bl. 74 und 76-81. Zum Verhältnis von Zentral- und Landesverwaltungen bei der Wirtschaftsplanung: Steiner, Zwischen Länderpartikularismus und Zentralismus, S. 34-37.

${ }^{99}$ SAPMO, NY 4182/963, Bl. 196-198, Bruno Leuschner am 4. 6. 1948 an Heinrich Rau.

100 SAPMO, DY 30/IV 2/2.1/206, stenographische Niederschrift der Sitzung des Zentralsekretariats am 10. 6. 1948.

101 SAPMO, NY 4182/963, Bl. 40f., Ergänzungsvorschläge der Abt. Arbeit und Sozialfürsorge beim SED-Zentralsekretariat vom 8.7. 1948 zum Entwurf über den Arbeitsplan für die Kampagne über den Zweijahrsplan 1949/50.

102 SAPMO, NY 4182/963, Bl. 89-91. 
erübrige sich - so Leuschner weiter - die Existenz der Hauptverwaltung Wirtschaftsplanung ${ }^{103}$. Offensichtlich war es dem stellvertretenden Leiter der Hauptverwaltung Kohle gelungen, eine Reduzierung der Produktionszahlen in Karlshorst zu erreichen, um auf diese Weise früher in den Genuß von Prämienzahlungen zu gelangen, die bei Übererfüllung von Planvorgaben ausgezahlt wurden. Dieser Schritt war mit dem Leiter der DWK-Hauptverwaltung Wirtschaftsplanung Leuschner nicht abgestimmt worden, der sich übergangen fühlte.

Für die weitere Einbettung der Arbeitskräftelenkung in die im Aufbau befindliche Planwirtschaft der SBZ/DDR hatten der Halbjahrsplan 1948 sowie der bereits angesprochene Zweijahrplan 1949/50 eine herausragende Bedeutung ${ }^{104}$. Darüber hinaus war mit der Gründung der DWK 1947 eine zentrale Instanz geschaffen worden ${ }^{105}$, die zusammen mit den zuständigen Hauptverwaltungen die wirtschaftspolitische Koordinierungsfunktion alleine für sich beanspruchte, wenn auch in enger Abstimmung mit der SED-Führung. Damit waren die institutionellen Voraussetzungen in Richtung Zentralisierung weitgehend gelegt worden. Eine weitere Folgewirkung dieser Maßnahmen bestand in der Zurückdrängung des Einflusses der Länder, die kaum noch über Mitsprache- und Gestaltungsmöglichkeiten verfügten. Für die Lenkung des Produktionsfaktors ,Arbeit' bleibt festzuhalten, daß zunächst einmal die Frage des strukturellen Aufbaus der Arbeitsverwaltung endgültig entschieden wurde. Des weiteren wandelte sich die Planungstätigkeit: Die Aufstellung eines Wirtschaftsplanes für die SBZ zog die Planung des Arbeitskräfteeinsatzes durch die HVAS zwingend nach sich. Damit verloren die Monats- und Quartalsberichte der deutschen Arbeitsverwaltung ihre ursprüngliche Bedeutung. Sie dienten ab sofort nicht mehr ausschließlich nur zur Weitergabe von Informationen an die sowjetische Besatzungsmacht sowie an andere DWK-Hauptverwaltungen, sondern besaßen nunmehr richtungsweisende Funktion für die nachgeordneten Verwaltungen auf Landes- bzw. Kommunalebene. Die nächste Weichenstellung sollte dann Anfang der fünfziger Jahre mit dem ersten Fünfjahrplan erfolgen: Der dadurch massiv einsetzende Auf- und Ausbau der Schwerindustrie ${ }^{106}$ legte auch für die Arbeitsverwaltung die Schwerpunkte erneut fest. Die Gewinnung von Arbeitskräften für den Bergbau, die Hüttenindustrie, den Schwermaschinenbau sowie den Allgemeinen Maschinenbau hatte somit eindeutig Priorität gewonnen.

Die SED-Führung beobachtete sehr genau die Durchführung des Zweijahrplanes durch die Arbeitsverwaltung, die auch der SMAD-Abteilung Arbeitskraft gegenüber zu Rechenschaft verpflichtet war ${ }^{107}$. Der Leiter der Hauptabteilung Arbeitskräfte bei der DWK-Hauptverwaltung Wirtschaftsplanung Mühlberg

${ }^{103}$ Ebenda, Bl. 196-198, hier Bl. 197, Leuschner am 4.6. 1948 an den DWK-Vorsitzenden Heinrich Rau.

104 Vgl. Staritz, Die Gründung der DDR, S. 140f.; Weber, Geschichte der DDR, S. 170-172.

105 Vgl. Steiner, Zwischen Länderpartikularismus und Zentralismus; Zank, Wirtschaftliche Zentralverwaltungen, in: SBZ.-Handbuch, S. 253-290.

$1 \% 6$ Roesler, Die Herausbildung der sozialistischen Planwirtschaft, S. 20 f.; Wienert, Die Stahlindustric in der DDR, S. 28-33.

107 Vgl. BAB, DQ 2/1950, Bericht über Besprechung bei der SMAD am 3. 8. 1948; BAB, DQ 2/2040, Bl. 162, Aktennotiz über Besprechung bei der SMAD in Karlshorst am 1. 10. 1948; BAB, DQ 2/ 1948, Niederschrift über Besprechung bei der SMAD in Karlshorst am 23. 11. 1948. 
zeichnete ein relativ düsteres Bild über den Zustand der Erwerbsbevölkerung in der SBZ. Im einzelnen wies er auf ein Absinken des Anteils der Erwerbsfähigen an der Gesamtbevölkerung (1939: 62 Prozent; 1946: 56 Prozent) und damit auf eine stetig wachsende Überalterung hin ${ }^{108}$. Darüber hinaus war nach seinen Angaben das Arbeitskräftepotential weitgehend ausgeschöpft: „Aus der zukünftigen Entwicklung 1949/50 der Bevölkerungszahl sind keine nennenswerten Erhöhungen (höchstens 50-60000) der Arbeitskräfte zu erwarten." 109 Bei einer Besprechung mit den Abteilungsleitern des SED-Zentralsekretariats zeigte sich Walter Ulbricht am 24. August 1948 sehr unzufrieden über die Tätigkeit der HVAS. Diese beschäftige sich - so der stellvertretende SED-Vorsitzende - „nicht ernsthaft mit der Beschaffung der Arbeitskräfte". Er sprach sich sogar für eine deutliche Reduzierung des Personalbestandes der Hauptverwaltung sowie für eine Reorganisation durch die DVdI aus ${ }^{110}$. Paul Merker griff diesen Gedanken einige Monate später wieder auf und arbeitete eine entsprechende Vorlage für das Zentralsekretariat aus. Seinen Angaben zufolge war das Personal bei den Arbeitsämtern nicht gleichmäßig auf die einzelnen arbeitsmarktpolitischen Bereiche aufgeteilt worden. Eine Änderung konnte - so Merker - nur von der SED-Führung direkt durchgesetzt werden: Um eine „durchgreifende Reorganisation der Abteilungen zu erreichen, ist ein zentrales Eingreifen notwendig "111. Eine Kommission, bestehend aus drei leitenden Mitarbeitern der HVAS, einem FDGB-Vertreter und einem Mitarbeiter der Abteilung Arbeit und Sozialfürsorge beim SED-Zentralsekretariat, sollte die einzelnen Landesämter überprüfen und die Neuordnung der Verwaltungsstrukturen vornehmen.

Der bis zur Gründung der DDR führende SED-Sozialpolitiker Helmut Lehmann hatte bereits bei der Ausarbeitung des Zweijahrplanes ein ganzes Maßnahmenbündel zur Deckung des auftretenden Arbeitskräftebedarfs vorgeschlagen $^{112}$. Zeitliche Verzögerungen sowie Reibungsverluste bei der Durchführung der Planungsvorgaben sollten auf diese Weise vermieden werden. So sollten die Betriebe vor einer Vermittlung von Arbeitskräften zunächst „auf die Berechtigung der Anforderung “ überprüft werden. Es war beabsichtigt, die Hortung von Arbeitskräften in den wirtschaftlich durch Materialengpässe und Kapitalknappheit weitgehend lahmgelegten Betrieben zu verhindern oder zumindest zurückzudrängen. Offen blieb jedoch die Frage, inwieweit die Arbeitsverwaltung noch einen Schritt weiter gehen und eine Auskämmungsaktion starten sollte. Lehmann sprach sich für die innerbetriebliche „Umsetzung" von Arbeitern aus: Demzufolge waren Arbeitsplätze „für leichtere Arbeit mit Frauen zu besetzen." Der Arbeitskräftebestand sollte insgesamt vergrößert werden. Neben den Frauen nannte Lehmann vor allem Kriegsheimkehrer und arbeitsuchende Schwerbeschädigte als

${ }^{10 *}$ BAB, DQ 2/1948, Notiz Mühlbergs vom 17. 8. 1948, S. 1.

109 Ebenda, S. 2. In einer Denkschrift vom 11. 2. 1949 machte Mühlberg erneut auf die Überalterung der Erwerbsbevölkerung sowie den bevorstehenden Arbeitskräftemangel aufmerksam. BAB, DC 15/59, Bl. 2-7, hier Bl. 1 .

110 SAPMO, DY 30/IV 2/2.027/2, Bl. 47-49, hier Bl. 48.

111 SAPMO, DY 30/IV 2/2.022/16, Bl. 345, Vorlage Merkers vom 14. 1. 1949.

112 SAPMO, DY 30/IV 2/2.022/21, Bl. 37f., Vorlage Lehmanns vom 13. 9. 1948 für das SED-Zentralsekretariat. 
potentielle Arbeitskräfte113. Von zentraler Bedeutung erschien aber die Verbesserung der Berufsumschulung "von Angehörigen überfüllter Berufe" und die "Nachwuchsplanung für die Berufsausbildung aller Jugendlichen" 114 . Dazu sollten im einzelnen Lehrwerkstätten und Berufsschulen weiter ausgebaut und die Berufsberatung intensiviert werden. Während die Berufsausbildung konzeptionell sehr früh mit der Arbeitskräftelenkung eng verbunden war, kann dies für die Lohn- und Wohnungsbaupolitik nur bedingt gesagt werden. Beides hatte zwar eine Anreizfunktion und sollte die Binnenwanderung hin zu den industriellen Schwerpunkten steuern. Dies war allerdings nur eine Funktion; daneben spielten andere Faktoren ebenfalls eine herausragende Bedeutung, wie bereits gezeigt werden konnte.

Der institutionelle Umbau des staatlichen Lenkungsapparates war im Herbst 1948 noch lange nicht abgeschlossen. Die Zentralisierung war aus Sicht der SEDFührung offensichtlich noch nicht weit genug gegangen. Dies hing vor allem damit zusammen, daß die einzelnen Hauptverwaltungen immer noch über genügend Gestaltungsspielräume verfügten, die eine schnelle und effiziente Planung erschwerten. So sprach sich etwa der Leiter der Hauptverwaltung Wirtschaftsplanung dafür aus, die von ihm geführte Hauptverwaltung "aus dem Rahmen der operativen Hauptverwaltungen herauszunehmen“ und zu dem „zentralen Leitungs- und Koordinierungsorgan für alle Planungsfragen “ in der SBZ aufzuwerten ${ }^{115}$. Nicht nur die anderen Hauptverwaltungen, sondern auch die zuständigen Ministerien auf Landesebene wären somit weisungsabhängig geworden. Nach Auffassung Leuschners sollte die Hauptverwaltung für Wirtschaftsplanung in „Zentralamt für Planung“ oder „Zentrales Planungsamt" umbenannt werden ${ }^{116}$. Dieser Vorstoß wurde jedoch nicht aufgegriffen und verhallte zunächst einmal.

Im Zusammenhang mit dem Zweijahrplan entwickelte sich eine Diskussion über die zukünftigen Aufgaben der Arbeitsämter, an der sich neben der HVAS die einzelnen Landesämter sowie die SED-Führung beteiligten. Langfristig betrachtet ging es um das Ziel, von einer reinen Arbeitsvermittlung hin zu einer Arbeitskräfteplanung zu gelangen ${ }^{117}$. Dazu sollten die Arbeitsämter strukturell und personell umgebaut werden. Letztlich bedeutete dieses Vorhaben ein Zurückdrängen der Arbeitsverwaltung bzw. eine stärkere institutionelle Einbettung in die allgemeine Wirtschaftsplanung und vor allem eine Reduzierung des Personalbestandes in den Arbeitsämtern ${ }^{118}$. Dagegen protestierte wiederum eine Reihe von Amtsleitern ${ }^{119}$. Hier deutete sich aber insgesamt ein Funktionswandel der Arbeitsverwaltung an, der 1951 seinen vorläufigen Abschluß finden sollte. Vorläufig bleibt festzuhalten, daß selbst die HVAS-Leitung mit dieser inhaltlichen Abwertung der Arbeitsämter im großen und ganzen einverstanden war.

113 Vgl. dazu im einzelnen Boldorf, Eingliederung der Kriegsopfer und Schwerbeschädigten.

$11+$ SAPMO, DY 30/IV 2/2.022/21, Bl. 38, Vorlage Lehmanns vom 13. 9. 1948 für das SED-Zentralsekretariat.

115 SAPMO, NY 4182/963, Bl. 219-221, hier Bl. 219, Leuschner am 12. 10. 1948 an Heinrich Rau.

116 Ebenda, Bl. 220.

$117 \mathrm{BAB}, \mathrm{DQ} 221763$, Protokoll über die Arbeitsamtsleitertagung in Potsdam am 20.10. 1948.

$118 \mathrm{Vgl}$. Max Herm: Zur Durchführung des Zweijahrplanes, in: Arbeit und Sozialfürsorge 3 (1948), S. $277 \mathrm{f}$

119 BAB, DQ 2/1763, Protokoll über die Arbeitsamtsleitertagung in Potsdam am 20.10. 1948, S. 1. 
Im weiteren Verlauf der Planungsarbeiten kristallisierte sich immer stärker heraus, daß eine gleichmäßige Steuerung des Arbeitskräftepotentials mit dem Ziel einer vollständigen Bedarfsdeckung nicht zu verwirklichen war. Die HVAS sowie die Hauptverwaltung Wirtschaftsplanung konzentrierten sich deshalb darauf, zumindest den Arbeitskräftebedarf in den Schwerpunktbetrieben (Volkseigene und SAG-Betriebe) zu befriedigen 120. Damit kehrten die Planungsbehörden zu der ursprünglichen Politik weitgehend zurück. Das Konzept, innerhalb der allgemeinen Wirtschaftsplanung die Zuteilung der Arbeitskräfte umfassend und vor allem effizient vornehmen zu können, erwies sich angesichts der nach wie vor bestehenden Mangel- und Besatzungssituation (Reparationen) vorerst noch als Illusion. Da die Anforderungen der Volkseigenen Betriebe sowie der SAG-Betriebe nicht abrissen, sah sich die DWK und die Arbeitsverwaltung letztlich gezwungen, eine pragmatische Arbeitsmarktpolitik zu betreiben. Bei der Versorgung der Schwerpunktbetriebe mit Arbeitskräften konnte die HVAS im übrigen partielle Erfolge vorweisen. Das betraf allerdings in erster Linie die Bereitstellung von Hilfsarbeitern, während die Nachfrage nach Facharbeitern nach wie vor nicht abgedeckt werden konnte ${ }^{121}$. Die HVAS beabsichtigte im Frühjahr 1949, Betriebsprüfungen durch die Arbeitsämter vornehmen zu lassen, um dadurch einen Überblick über die „Zweckmäßige Verwendung und Auslastung des wichtigen Produktionsfaktors ,menschliche Arbeitskraft" " zu gewinnen. Dies war aus Sicht der Berliner Hauptverwaltung „eine wesentliche Vorbedingung für die Erfüllung der Planaufgaben"122. Der Erfolg dieser Aktion war ganz entscheidend abhängig von der Bereitschaft der Betriebsleitungen zur Mitarbeit; darüber liegen jedoch keine Angaben vor. Das Sekretariat der DWK verabschiedete auf seiner Sitzung am 14. September 1949 eine Anordnung „über die bevorzugte Versorgung der Schwerpunktbetriebe des Maschinenbaus und der Metallurgie mit Material, Arbeitskräften und Transportmitteln"123, die offensichtlich der Leiter des Fachsekretariats Industrie, Fritz Selbmann, auf Befehl der SMAD eingebracht hatte ${ }^{124}$. Meinungsverschiedenheiten zwischen einzelnen Hauptverwaltungen über die endgültige Formulierung von $\$ 4125$ sowie die Aufstellung der Liste mit den Schwerpunktbetrieben ${ }^{126}$ verzögerten den Abschluß.

120 BAB, DQ 2/1764, Bl. 23-27, Denkschrift der HVAS (o.D.) über die Aufgaben der Arbeitsämter "auf dem Gebiete der Arbeitskraftlenkung zur Erfüllung des Zweijahrplanes“.

121 BAB, DQ 2/2063, Niederschrift über Besprechung der HVAS-Abteilungen I a und I b am 3.5. 1949, S. 2.

122 BAB, DQ 2/2063, Richtlinienentwurf der HVAS-Abt. I a vom 2. 6. 1949, S. 1.

123 BAB, DC $15 / 420$, Bl. 2.

${ }^{124}$ BAB, DC 15/II/1-42, B1. 81, Selbmann am 7.9. 1949 an das Sekretariat der DWK (Schaul). Der Anordnungsentwurf befindet sich in: ebenda, Bl. 69-72.

125 Dieser berechtigte die Schwerpunktbetriebe, in den Handelszentralen sowie in den Fachgebieten und Fachkontoren der Deutschen Handelsgesellschaft Roh- und Hilfsstoffe ohne besondere Freigabe einzukaufen. Ebenda, Bl. 70. Die endgültige Fassung von $\$ 4$ enthielt allerdings ein Genehmigungsrecht der Hauptverwaltung Materialversorgung. Ebenda, Bl. 75.

126 In der Anlage zum Beschluß wurden insgesamt 41 Maschinenbaubetriebe aufgeführt. Ebenda, Bl. 77. 